\title{
Cancelamento das Forças de Vínculo em Anel Condutor Carregado com $n q$ Cargas por Meio de uma Carga $Q$ de Sinal Contrário Posicionada no Centro
}

Cancellation of constraint forces in the charged conductor ring with $n q$ charges by a charge $Q$ with opposite sign placed on the center

\author{
Norberto Helil Pasqua* e Paulo Daniel Emmel ${ }^{\dagger}$ \\ Departamento de Física, Universidade Federal de São Carlos \\ UFSCar (Universidade Federal de São Carlos) - Brasil \\ C.P. 676, 13565-905, São Carlos, SP, Brasil
}

Recebido em 04/01/2001. Aceito em 12/03/2001

\begin{abstract}
O problema proposto é o de um anel condutor de raio $R$ carregado com $n$ cargas $q$, em que se deseja anular as forças de vínculo que nele atuam. A solução é colocar no centro do anel determinada carga $Q$ de sinal contrário, de modo que a soma das forças elétricas sobre cada carga do anel devido à carga central e às outras cargas do anel seja nula. Busca-se, portanto, uma função $Q(n q)$ que forneça o valor da carga central em função de $n q$. O resultado mostra-nos que $Q(n q)$ é proporcional a $n \ln (n) q$ para $n \gg 2$.
\end{abstract}

The proposed problem is to calculate the charge to be placed in the center of a ring, in which we distribute $n$ charges equal to $q$, so that the constraint force become null. The solution for this problem is to place a charge $Q$ of opposite sign in the center of the ring, so the sum of electric forces in each charge in the ring, due to the central charge and the other charges, become null. We search for a function $Q(n q)$ that gives the value of $Q$ in terms of $n q$. The result is that $Q(n q)$ is proportional to $n \ln (n) q$ for $n \gg 2$.

\section{Introdução}

Consideremos um anel condutor de raio $R$, cujo fio tenha um diâmetro tal que qualquer quantidade de cargas $(n \geqslant 2)$ seja distribuída linearmente em toda sua extensão. Tomando-se $n q$ cargas idênticas entre si e abandonando-as aleatoriamente nesse anel, observar-seá o movimento dessas até encontrarem posições em que não experimentem nenhuma força resultante.

O movimento observado da $i$-ésima carga - até o estabelecimento do equilíbrio eletrostático - é devido à força repulsiva de Coulomb [1] entre esta e as demais. Esta força tem a forma

$$
\mathbf{F}_{i}=\frac{q_{i}}{4 \pi \epsilon_{0}} \sum_{j}^{n}, \frac{q_{j}}{r_{i j}^{2}} \hat{\mathbf{r}}_{i j},
$$

onde $r_{i j}=\left|\mathbf{r}_{i}-\mathbf{r}_{j}\right|$ é a distância entre duas cargas, $\epsilon_{0}$ é constante de permissividade elétrica e no somatório não se considera $j=i$.

Apesar da força repulsiva, as cargas permanecerão no anel devido à presença de forças de vínculo, que também são de origem elétrica. Conseqüentemente, quanto maior $n$, maior deverá ser a intensidade de tais forças para que o sistema permaneça em equilíbrio. $\mathrm{O}$ acúmulo de cargas no anel gera tensões que podem ser anuladas, colocando-se determinada carga puntiforme $Q$ de sinal oposto a $q$ posicionada no centro do anel. Anular essas forças de vínculo é equivalente a obter uma força resultante nula, $\mathbf{F}_{\text {res }}=\mathbf{0}$, sobre cada carga do sistema, isto é, sobre as $n q$ cargas do anel e sobre a carga central $Q$.

Para o cálculo de $Q(n q)$, considerou-se um sistema cartesiano (vide Figuras 1, 2 e 3 ) com origem em uma carga $q_{1}, P_{q_{1}}(0,0)$, de modo que o eixo-y tangencie o anel e o eixo- $x$ passe pelo seu centro, onde está posicionada a carga central, $P_{Q}=(R, 0)$. Dada a simetria do anel e o mesmo valor das $n$ cargas, o ângulo formado pelos raios de duas cargas consecutivas será de $2 \pi / n$. Já a distância em módulo de $q_{1}$ até a $j$-ésima carga $q_{j}$ projetada sobre o eixo- $x$ pode ser escrita como $d\left(q_{1}, q_{j}\right)=$ $\left|\mathbf{r}_{1 j}\right|=2 R \cos \alpha_{1 j}, \alpha_{1 j}=-\left[1-\frac{2}{n}(j-1)\right] \frac{\pi}{2}$ o ângulo

\footnotetext{
*e-mail: (pasqua ou g167460)@polvo.ufscar.br

†e-mail: emmel@power.ufscar.br
} 
formado entre $\mathbf{r}_{1 j}$ e o eixo- $x, \operatorname{com} j=2,3, \ldots, n$. Observe-se que, por construção, $\alpha_{1 j}$ encontra-se no intervalo $\left(-\frac{\pi}{2}, \frac{\pi}{2}\right)$, não variando, portanto, o sinal de $\cos \alpha_{1 j}$, que será sempre positivo. A construção do sistema dessa forma apresenta a vantagem da simplificação do cálculo, uma vez que, dada a simetria da distribuição, a componente da força resultante paralela ao eixo- $y$ será sempre nula, e a componente paralela ao eixo- $x$ tomada sobre a carga $q_{1}$, terá a forma

$$
F_{1 x}=\left|\mathbf{F}_{1 Q}\right|-\sum_{j=2}^{n}\left|\mathbf{F}_{1 j}\right| \cos \alpha_{1 j}=0
$$

\section{Desenvolvimento}

Consideremos primeiramente $n=2$, ou seja, duas cargas $q$ sobre o anel. Procura-se por um determinado valor de $Q(2 q)$ tal que $\mathbf{F}_{\text {res }}=\mathbf{0}$. No equilíbrio, as duas cargas $q_{1}$ e $q_{2}$ encontram-se diametralmente opostas $\left(\alpha_{12}=0\right)$. Do ponto de vista de $q_{1}$, ou seja, a partir de $q_{1}$, obtem-se

$$
F_{1 x}=\left|\mathbf{F}_{1 Q}\right|-\left|\mathbf{F}_{12}\right|=0
$$

$\mathrm{Ou}$

$$
\frac{\left|q_{1}\right||Q|}{r_{1 Q}^{2}}=\frac{q_{1} q_{2}}{r_{12}^{2}} .
$$

Sendo $\left|\mathbf{r}_{1 Q}\right|=R,\left|\mathbf{r}_{12}\right|=2 R$, e $q_{1}=q_{2}=q$, tem-se que

$$
Q(2 q)=\frac{q}{4}
$$

Note-se que $Q(2 q)$ não depende do raio do anel. Obviamente, isto deve-se ao fato de $Q$ estar posicionada a meia distância entre $q_{1}$ e $q_{2}$.

Tomemos, agora, três cargas $q$ sobre o anel e usando como referência a Figura 1, calculemos o valor de $Q(3 q)$ de maneira que a resultante na direção $\hat{\mathbf{x}}$ seja nula,

$$
F_{1 x}=\left|\mathbf{F}_{1 Q}\right|-\left|\mathbf{F}_{12}\right| \cos \alpha_{12}-\left|\mathbf{F}_{13}\right| \cos \alpha_{13}=0 .
$$

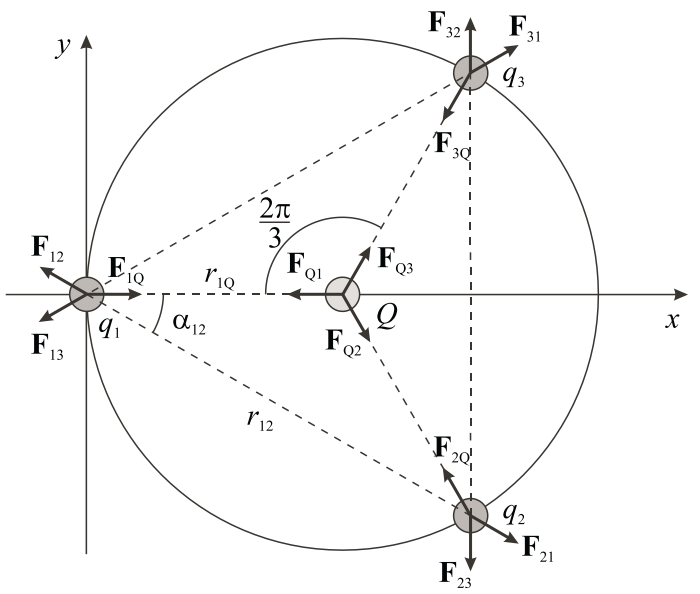

Figura 1. Três cargas distribuídas sobre o anel condutor.
Como $\left|\mathbf{F}_{12}\right|=\left|\mathbf{F}_{13}\right|, q_{1}=q_{2}=q,\left|\mathbf{r}_{1 Q}\right|=R \mathrm{e}$ $\left|\mathbf{r}_{12}\right|=\left|\mathbf{r}_{13}\right|=2 R \cos \alpha_{12}$, sendo $-\alpha_{12}=\alpha_{13}=\frac{\pi}{6}$, segue que

$$
\frac{|Q|}{R^{2}}=2 \frac{q}{\left(2 R \operatorname{sen} \frac{\pi}{3}\right)^{2}} \operatorname{sen} \frac{\pi}{3},
$$

pois $\cos \left(\frac{\pi}{2}-\theta\right)=\operatorname{sen} \theta$. Aqui, para $n=3$, novamente $Q$ não depende de $R$ como era de se esperar. Finalmente,

$$
Q(3 q)=\frac{q}{2 \operatorname{sen} \frac{\pi}{3}}
$$

Para quatro cargas $q$ sobre o anel, a componente $x$ da força resultante tem a seguinte forma

$F_{1 x}=\left|\mathbf{F}_{1 Q}\right|-\left|\mathbf{F}_{12}\right| \cos \alpha_{12}-\left|\mathbf{F}_{13}\right|-\left|\mathbf{F}_{14}\right| \cos \alpha_{14}=0$

A partir da Figura 2, observa-se que,

$$
\frac{|Q|}{R^{2}}=2 \frac{q}{\left(2 R \cos \alpha_{12}\right)^{2}} \cos \alpha_{12}+\frac{q}{(2 R)^{2}},
$$

$\mathrm{Ou}$

$$
Q(4 q)=\frac{q}{2}\left(\frac{1}{\operatorname{sen} \frac{\pi}{4}}+\frac{1}{2}\right)
$$

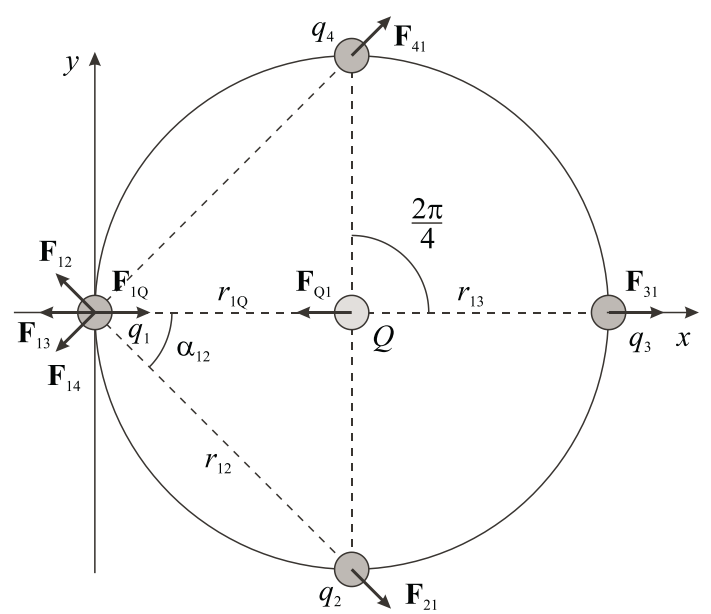

Figura 2. Quatro cargas distribuídas sobre o anel condutor.

Ainda é insuficiente o número de cargas para que se possa abstrair e obter uma função. Sigamos em frente acrescentando mais cargas ao anel.

Tomemos cinco cargas $q$ sobre o anel $(n=5)$, conforme Figura 3. 


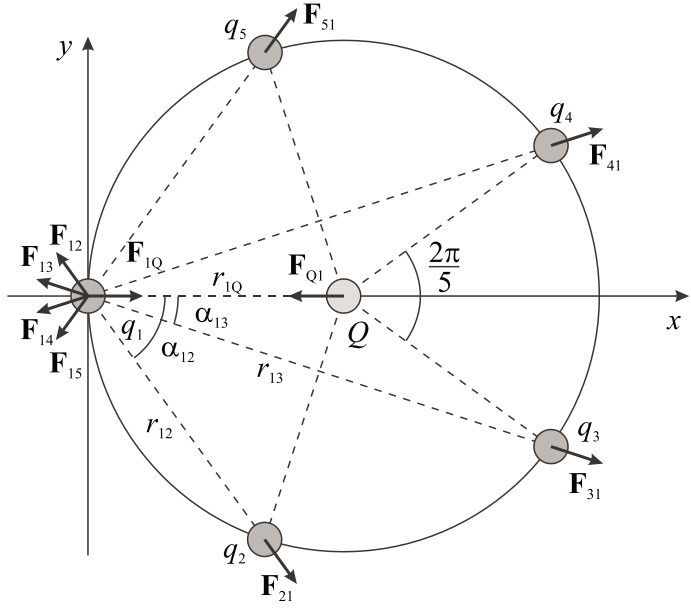

Figura 3. Cinco cargas distribuídas sobre o anel condutor.

Então,

$$
F_{1 x}=\left|\mathbf{F}_{1 Q}\right|-\left|\mathbf{F}_{12}\right| \cos \alpha_{12}-\left|\mathbf{F}_{13}\right| \cos \alpha_{13}
$$

- Duas cargas $q \Rightarrow Q(2 q)=\frac{q}{2}\left[\frac{1}{2 \operatorname{sen}\left(\frac{\pi}{2}\right)}\right]$

- Três cargas $q \Rightarrow Q(3 q)=\frac{q}{2}\left[\frac{1}{\operatorname{sen}\left(\frac{\pi}{3}\right)}\right]$

- Quatro cargas $q \Rightarrow Q(4 q)=\frac{q}{2}\left[\frac{1}{\operatorname{sen}\left(\frac{\pi}{4}\right)}+\frac{1}{2 \operatorname{sen}\left(\frac{\pi}{4} 2\right)}\right]$

- Cinco cargas $q \Rightarrow Q(5 q)=\frac{q}{2}\left[\frac{1}{\operatorname{sen}\left(\frac{\pi}{5}\right)}+\frac{1}{\operatorname{sen}\left(\frac{\pi}{5} 2\right)}\right]$

- Seis cargas $q \Rightarrow Q(6 q)=\frac{q}{2}\left[\frac{1}{\operatorname{sen}\left(\frac{\pi}{6}\right)}+\frac{1}{\operatorname{sen}\left(\frac{\pi}{6} 2\right)}+\frac{1}{2 \operatorname{sen}\left(\frac{\pi}{6} 3\right)}\right]$

- Sete cargas $q \Rightarrow Q(7 q)=\frac{q}{2}\left[\frac{1}{\operatorname{sen}\left(\frac{\pi}{7}\right)}+\frac{1}{\operatorname{sen}\left(\frac{\pi}{7} 2\right)}+\frac{1}{\operatorname{sen}\left(\frac{\pi}{7} 3\right)}\right]$

- Oito cargas $q \Rightarrow Q(8 q)=\frac{q}{2}\left[\frac{1}{\operatorname{sen}\left(\frac{\pi}{8}\right)}+\frac{1}{\operatorname{sen}\left(\frac{\pi}{8} 2\right)}+\frac{1}{\operatorname{sen}\left(\frac{\pi}{8} 3\right)}+\frac{1}{2 \operatorname{sen}\left(\frac{\pi}{8} 4\right)}\right]$

$$
-\left|\mathbf{F}_{14}\right| \cos \alpha_{14}-\left|\mathbf{F}_{15}\right| \cos \alpha_{15}=0,
$$

$\mathrm{ou}$

$$
\frac{|Q|}{R^{2}}=2 \frac{q}{\left(2 R \cos \alpha_{12}\right)^{2}} \cos \alpha_{12}+2 \frac{q}{\left(2 R \cos \alpha_{13}\right)^{2}} \cos \alpha_{13} .
$$

Portanto,

$$
Q(5 q)=\frac{q}{2}\left(\frac{1}{\operatorname{sen} \frac{\pi}{5}}+\frac{1}{\operatorname{sen} \frac{\pi}{5} 2}\right)
$$

Seguindo o mesmo raciocínio para alguns outros valores de $n$ e organizando os dados como feito abaixo, verifica-se que há uma relação entre o número de cargas e o número de parcelas a serem somadas, bem como a variação do valor do argumento de seno:
Pode-se notar a existência de dois padrões, um para número par de cargas $q$ e, outro para número ímpar. É possível então generalizar, de modo a encontrar o valor de $Q(n q)$ para qualquer $n \geqslant 2$ :

$$
Q(n q)=\frac{q}{2} \cdot \sum_{i=1}^{\frac{n-1}{2}} \frac{1}{\operatorname{sen}\left(\frac{\pi}{n} i\right)}, \text { para } n \text { ímpar }
$$

e,

$$
Q(n q)=\frac{q}{2} \cdot\left[\frac{1}{2}+\sum_{i=1}^{\frac{n-2}{2}} \frac{1}{\operatorname{sen}\left(\frac{\pi}{n} i\right)}\right] \text {, para } n \text { par. }
$$

A limitação para o uso da série diz respeito à capacidade de cálculo do computador e ao tempo disponível. 
Para $n$ muito grande - cuja ordem de grandeza supere a precisão da máquina - o cálculo torna-se além de lento, impreciso. O grande número de termos somados torna o erro absoluto maior que o valor das parcelas adicionadas, prejudicando a obtenção do valor da carga central. Uma solução para este problema é fracionar a soma de modo a limitar a propagação do erro.

A Figura 4 abaixo mostra como evolui o valor da carga central em relação à carga distribuída sobre o anel condutor num intervalo de $n=2$ a 10 cargas (neste e nos demais gráficos a carga $q$ foi tomada igual à unidade). Este gráfico informa que o valor da carga central cresce mais rapidamente que a carga do anel.

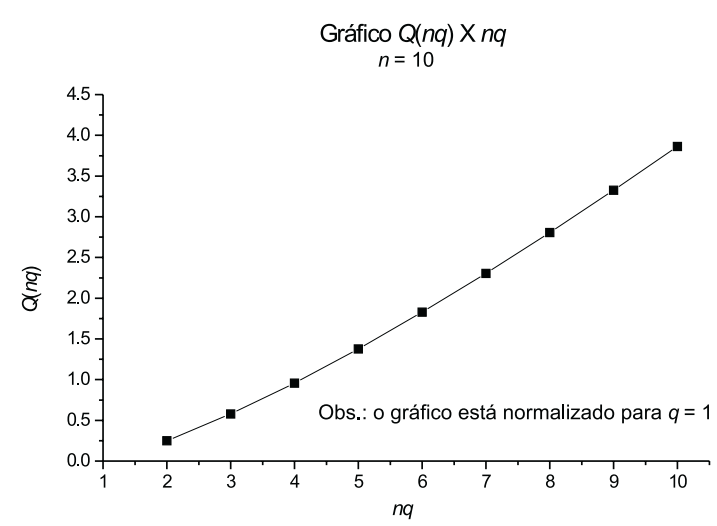

Figura 4. Evolução da série no intervalo $n=2 \ldots 10$.

Como se comporta $Q(n q)$ para valores grandes de $n$ ? A Figura 5 apresenta valores de $Q(n q)$ no intervalo de $n=2$ a $10^{6}$. Pode-se observar que, conforme $n$ cresce, a curva parece tender a uma reta. Pergunta-se: será $Q(n q)$ linear com $n q$ quando $n$ for muito grande? E, existe uma expressão matemática que descreve esse crescimento e que seja, ao mesmo tempo, mais prática que as séries encontradas acima? A resposta a ambas as perguntas é sim. O restante deste trabalho é dedicado à busca dessa expressão matemática e à sua análise.

A realidade física impõe que, para a detecção de cargas elétricas em um objeto, $n$ seja superior a $10^{6}$. Em eletrômetros típicos (como um Keithley modelo 617), o intervalo de operação encontra-se entre $10^{-13}$ e $10^{-8} \mathrm{~A}$. Sendo a carga elétrica elementar de um elétron ou de um próton equivalente a $1,602 \times 10^{-19} \mathrm{C}$, verifica-se que tais eletrômetros detectam quantidades de cargas entre $10^{6}$ e $10^{11}$ unidades. Tal intervalo será usado na obtenção e em análises de $Q(n q)$.

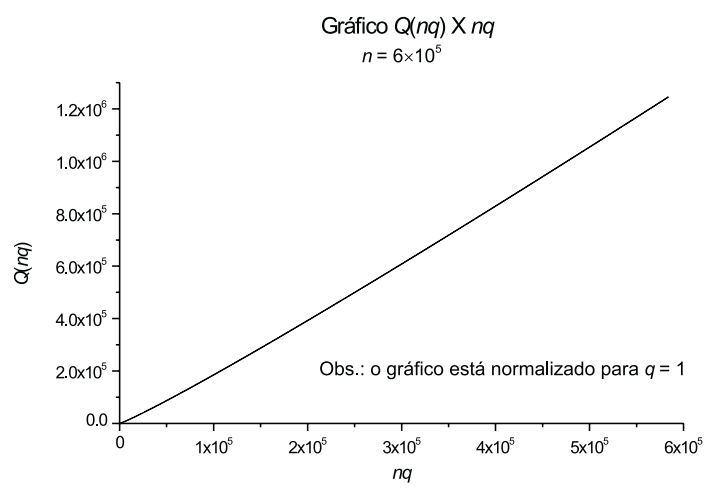

Figura 5. Evolução da série no intervalo $n=2 \ldots 10^{6}$.

Pode-se considerar a Eq.(7) e a Eq.(8) como sendo equivalentes, pois no intervalo citado acima é completamente indiferente $n$ ser par ou ímpar. Além disso, na Eq.(7) observa-se que o fator $1 / 2$ só tem importância quando $n \sim 10$, de modo que se pode desprezá-lo. Isto permite trabalhar com uma única expressão para $n$ grande. Então, tomando a série ímpar, $Q(n q)_{s r}$, tem-se

$$
Q(n q)_{s r}=\frac{q}{2} \cdot \sum_{i=1}^{\frac{n-1}{2}} \frac{1}{\sin \left(\frac{\pi}{n} i\right)} .
$$

Como a Eq.(9) não depende do raio do anel, este pode ser considerado de um tamanho tal que a distribuição de cargas seja contínua. Neste caso é fácil transformar a série ímpar em uma integral, usando a definição de soma de Riemann [2] para partição regular

$$
\lim _{\Delta x \rightarrow 0} \sum_{k} f\left(w_{k}\right) \Delta x=\int_{a}^{b} f(x) d x .
$$

Assim, fazendo na Eq.(9) $\frac{\pi}{n} i=x, \frac{\pi}{n}=\Delta x$ e tomando o limite de $\Delta x \rightarrow 0$, encontra-se

$$
Q(n q)=\frac{n}{\pi} \frac{q}{2} \cdot \int_{\frac{\pi}{n}}^{\frac{\pi}{2}-\frac{\pi}{2 n}} \csc x d x, \text { para } n \gg 2 .
$$

Após alguma manipulação algébrica obtem-se a seguinte função denominada obtida, $Q(n q)_{o b}$,

$$
Q(n q)_{o b}=\frac{n q}{2 \pi} \cdot \ln \left\{\frac{\cos \left(\frac{\pi}{2 n}\right)\left[1+\cos \left(\frac{\pi}{n}\right)\right]}{\left[1+\sin \left(\frac{\pi}{2 n}\right)\right] \sin \left(\frac{\pi}{n}\right)}\right\} .
$$

Comparando-se os gráficos das equações (9) e (10), verifica-se que, para valores grandes de $n$, essas parecem posicionar-se paralelamente (Figuras 6 e 7). É possível melhorar isto, visando a convergência das duas curvas, através de uma adequada alteração dos limites de integração. Tomaram-se, para tanto, intervalos de integração de tamanho constante e variou-se a origem do mesmo de modo a minimizar o valor absoluto da diferença entre a série e a integral. Uma boa aproximação 
foi conseguida através do seguinte intervalo: $x_{i}=\frac{9 \pi}{16} \mathrm{e}$ $x_{f}=\frac{\pi}{2}-\frac{15 \pi}{16 n}$, de modo a obter-se a função melhorada, $Q(n q)_{m e}$

$$
Q(n q)_{m e}=\frac{n q}{2 \pi} \cdot \ln \left\{\frac{\cos \left(\frac{15}{16} \frac{\pi}{n}\right)\left[1+\cos \left(\frac{9}{16} \frac{\pi}{n}\right)\right]}{\left[1+\sin \left(\frac{15}{16} \frac{\pi}{n}\right)\right] \sin \left(\frac{9}{16} \frac{\pi}{n}\right)}\right\}
$$

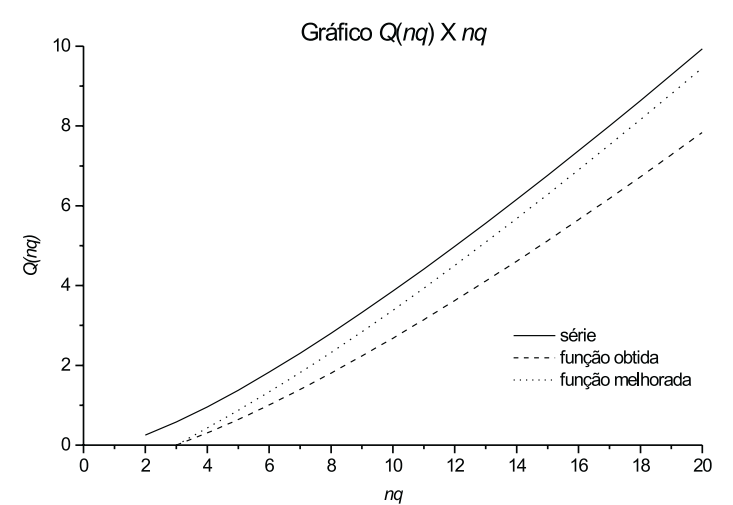

Figura 6. Comparação da série com as funções obtida e melhorada no intervalo $n=2 \ldots 20$. A legenda indica que a curva série refere-se à Eq.(9), função obtida com Eq.(10) e função melhorada em Eq.(11).

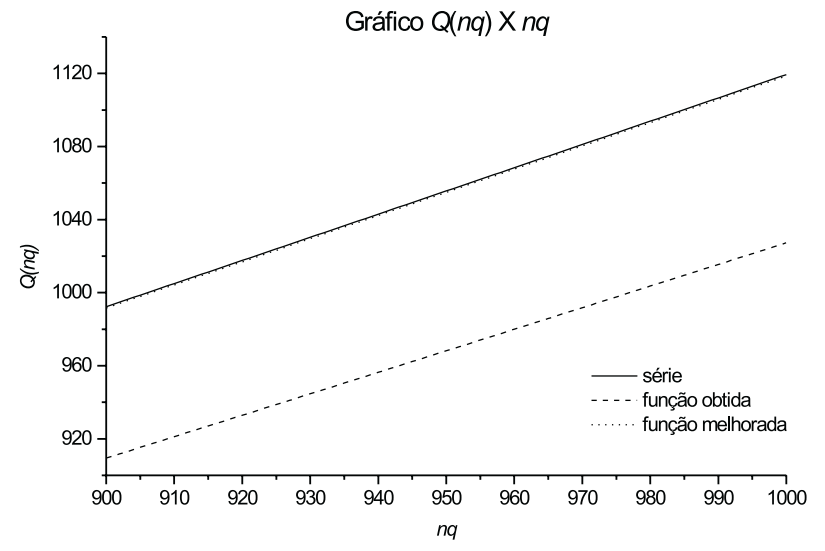

Figura 7. Gráfico comparativo da série com as funções obtida e melhorada no intervalo $n=900$ a 1000 cargas.

\begin{tabular}{|c|c|}
\hline $\mathbf{n}$ & função melhorada/série \\
\hline $10^{1}$ & 0,87557 \\
\hline $10^{2}$ & 0,99338 \\
\hline $10^{4}$ & 0.99977 \\
\hline $10^{8}$ & 0,99990 \\
\hline
\end{tabular}

Tabela 2 - A função melhorada converge assintoticamente para a série.
Pode-se notar que a função melhorada está de bom acordo com a série. Tal concordância, estudada para $n$ variando, em ordem, de $10^{1}$ a $10^{8}$, deu-se assintoticamente conforme observado na Tabela 2. Aqui é encontrada uma outra limitação para o cálculo de $Q$ através da série. A quantidade máxima de cargas permitida, ou o número inteiro máximo possível de se operar em linguagem Fortran 90 (for Windows) ${ }^{1}$ deve ser menor ou igual a $10^{9}$.

Devido à convergência da função melhorada é possível extrapolar os limites impostos ao uso da série. Para valores de $n$ com sentido físico, isto é, valores compreendidos entre $10^{6}$ e $10^{11}$ cargas elementares, a função representa uma boa ferramenta para o cálculo da carga central.

Pode-se obter uma função para o cálculo de $Q$ mais compacta, à medida em que se considera somente o termo dominante da Eq.(11). No intervalo entre $10^{6}$ e $10^{11}$ cargas elementares os argumentos de senos, de cosenos e de logaritmos neperianos são suficientemente pequenos para poderem ser expandidos em série de Taylor ${ }^{2}$ até termos de segunda ordem. Assim, a função aproximada, $Q(n q)_{a p}$, pode ser escrita aproximadamente como

$$
Q(n q)_{a p} \cong \frac{n q}{2 \pi} \cdot\left[\ln n+\ln \left(\frac{32}{9 \pi}\right)\right]-\frac{15}{32}+O\left(\frac{1}{n}\right) .
$$

Como $\frac{32}{9 \pi} \sim 1$ e a constante subtrativa pouco afeta o cálculo para o intervalo de $n$ considerado, pode-se finalmente escrever,

$$
Q(n q)_{a p}=\frac{q}{2 \pi} \cdot n \ln (n) .
$$

Deve-se observar, entretanto, que o termo dominante da série, Eq.(12) apresenta maior imprecisão que a Eq. (11) ou mesmo que a Eq.(10). No caso, como mostrado na Tabela 3, no intervalo de interesse, a imprecisão é cerca de uma parte em cem.

\begin{tabular}{|c|c|}
\hline$n$ & função aproximada / série \\
\hline $10^{1}$ & 0,94879 \\
\hline $10^{2}$ & 0,97345 \\
\hline $10^{4}$ & 0,98654 \\
\hline $10^{8}$ & 0,99323 \\
\hline
\end{tabular}

Tabela 3 - A função aproximada converge de forma mais lenta para a série, quando comparada com a função melhorada.

Embora $Q$ não seja linear com $n q$, conforme Eq.(12), tem-se que $\ln (Q)$ torna-se linear $\operatorname{com} \ln (n)$ para $n$ muito grande. Isto é verificado através da derivada logarítmica de $Q$

$$
\lim _{n \rightarrow \infty} \frac{\partial \ln \left[Q(n q)_{a p}\right]}{\partial \ln (n)}=\lim _{n \rightarrow \infty}\left[1+\frac{1}{\ln (n)}\right]=1,
$$

\footnotetext{
${ }^{1}$ No Fortran 90, o comando INTEGER(4) permite-nos operar com números inteiros no intervalo de -2.147 .483 .648 a 2.147.483.647. ${ }^{2} \cos (\theta) \cong 1-\frac{\theta^{2}}{2}+O\left(\theta^{4}\right), \sin (\theta) \cong \theta-O\left(\theta^{3}\right)$ e $\ln (1 \pm \theta) \cong \pm \theta-\frac{\theta^{2}}{2}+O\left(\theta^{3}\right)$
} 
e mostrado na Figura 8 através de um gráfico de $\frac{\partial \ln (Q)}{\partial \ln (n)}$ versus $\ln (n)$.

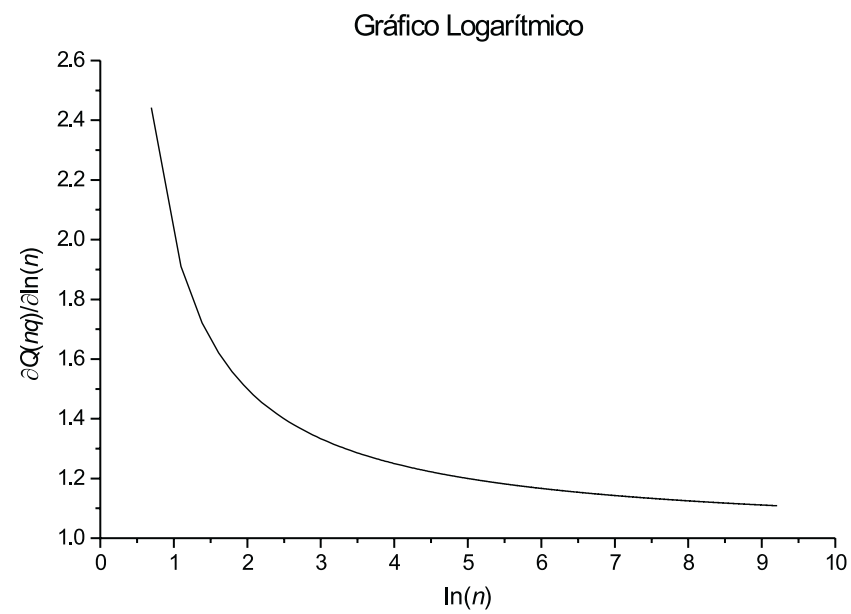

Figura 8. O valor de $\ln \left[Q(n q)_{m e}\right]$ tende a linearizar-se com $\ln (n)$ quando $n \gg 2$.

\section{Conclusão}

A função melhorada está de bom acordo com a série e, portanto, constitui um modo de se obter o valor da carga central de forma mais rápida. Com esta função, é possível extrapolar o valor da série em regiões onde o uso da série é impraticável por meios numéricos. Dentro da realidade física, isto é, com $n$ entre $10^{6}$ e $10^{11}$, pode-se usar a função aproximada (ou o termo dominante da série), isto é,

$$
Q(n q)_{a p}=\frac{q}{2 \pi} \cdot n \ln (n),
$$

o qual apresenta imprecisão de uma parte em cem nesse intervalo.

Cabe ressaltar que a proposta inicial deste estudo foi solucionar um problema essencialmente teórico. Permanece em aberto, portanto, o estudo de casos experimentais que venham a se utilizar do aqui exposto.

\section{Referências}

1. Reitz, J. R., Milford, F. J., Christy, R. W. Fundamentos da Teoria do Eletromagnetismo. $7^{a}$ edição, Rio de Janeiro, Campus, 1982.

2. Swokowski, E.W. Cálculo com Geometria Analítica. $2^{a}$ edição, São Paulo, Makron Books, 1994. 